\title{
Landau theory of domain wall magnetoelectricity
}

\author{
Maren Daraktchiev, ${ }^{1,2, *}$ Gustau Catalan, ${ }^{1,3, \dagger}$ and James F. Scott ${ }^{1,4, *}$ \\ ${ }^{1}$ Department of Earth Sciences, University of Cambridge, Downing Street, Cambridge CB2 3EQ, United Kingdom \\ ${ }^{2}$ Department of Health, 330 Wellington House, 133-155 Waterloo Road, London SE1 8UG, United Kingdom \\ ${ }^{3}$ ICREA and CIN2, CSIC-ICN, Campus Universitat Autonoma de Barcelona, Bellaterra 08193, Spain \\ ${ }^{4}$ Department of Physics, Cavendish Laboratory, University of Cambridge, Cambridge CB3 OHE, United Kingdom
}

(Received 21 February 2010; revised manuscript received 13 May 2010; published 30 June 2010)

\begin{abstract}
We calculate the exact analytical solution to the domain wall properties in a generic multiferroic system with two order parameters that are coupled biquadratically. This is then adapted to the case of a magnetoelectric multiferroic material such as $\mathrm{BiFeO}_{3}$, with a view to examine critically whether the domain walls can account for the enhancement of magnetization reported for thin films of this material, in view of the correlation between increasing magnetization and increasing volume fraction of domain walls as films become thinner. The present analysis can be generalized to describe a class of magnetoelectric devices based on domain walls rather than bulk properties.
\end{abstract}

DOI: 10.1103/PhysRevB.81.224118

PACS number(s): 77.80.Dj

\section{INTRODUCTION}

$\mathrm{BiFeO}_{3}$ was first researched in Leningrad in 1950 s and 1960s and has recently had a renaissance due to its roomtemperature magnetic and ferroelectric properties. ${ }^{1-5}$ The report by Wang ${ }^{1}$ of strong ferromagnetism in thin films of bismuth ferrite $\left(\mathrm{BiFeO}_{3}\right)$ has stimulated considerable interest in the possibility of using this material as a room-temperature magnetoelectric sensor or memory element. However, later studies have shown that the ferromagnetic moment is very small in other specimens, suggesting that the effect is not an intrinsic bulk symmetry property and may arise from oxygen vacancies, $\mathrm{Fe}^{+2}$ ions, or other point or extended defects. ${ }^{6-8}$ Domain walls are of course a special kind of extended defect, and we show here that they can, in principle, be ferromagnetic. This is a kind of magnetoelectricity that transcends the bulk symmetry properties and may be large enough to provide a different class of multiferroic device.

Beginning two decades ago, Lajzerowicz ${ }^{9}$ predicted that domain walls in systems with two coupled order parameters could lead to the emergence of one parameter inside the domain wall of the other. Transposing their results to the specific case of magnetoelectric multiferroics, the centers of domain walls could, in principle, bear out either a net electric $(P)$ or magnetic $(M)$ moment while the domain themselves were nonelectric $\left(P_{B}=0\right)$ or nonmagnetic $\left(M_{B}=0\right)$. This kind of ferroic behavior was generalized by Privratska and Janovec $^{10}$ in 1997 to show, using group-theoretical arguments, what crystal symmetries might exhibit it. The space group of $\mathrm{BiFeO}_{3}, R 3 c$, was one of them. Unfortunately, the Privratska-Janovec theory could not estimate magnitudes, or even whether the effect could be observable. Later Fiebig and co-workers also studied magnetoelectric effects in the domain walls of antiferromagnetic hexagonal manganites $\mathrm{YMnO}_{3}$ (Ref. 11) and $\mathrm{HoMnO}_{3} .{ }^{12}$ More recently, a linear correlation between the number density of (ferroelastic) domain walls and ferromagnetism has also been reported in thin films of $\mathrm{TbMnO}_{3},{ }^{13}$ suggesting that the ferroelastic walls of this antiferromagnetic material may be ferromagnetic. Finally, the converse effect of polarization emerging inside the domain walls of a magnet was beautifully demonstrated by Logginov et al. ${ }^{14}$

Taken together, then, all these findings indicate the possibility of having a class of magnetoelectric devices that we shall term "domain wall multiferroics." Although such domain wall effects may be small in bulk, a key point to bear in mind is that thin films have extremely small domains and thus a high volume density of domain walls ${ }^{15-17}$ so that the domain wall magnetoelectricity may play a very important role in thin-film devices. In the present work, we provide the analytical solution to the thermodynamic equations that describe the magnetoelectric coupling in the domain walls of a multiferroic system, and use the analysis to examine whether this effect may be behind the reported magnetization of $\mathrm{BiFeO}_{3}$ films.

Applications of free-energy models to multiferroic materials necessarily involve three coupled parameters: $M$, the net (weak) ferromagnetic magnetization, which usually arises from canting; $L$, the sublattice magnetization $(L>M \geq 0)$, and $P$, the ferroelectric polarization. Couplings of all three parameters generally need to be considered, as was done by Fox et al. ${ }^{18,19}$ for multiferroic $\mathrm{BaMnF}_{4}$. The magnetism in that material is a bulk effect directly correlated with the linear coupling of $L, M$, and $P .{ }^{18-20}$ By contrast, in the present paper, we try to see whether physical conclusions can be obtained via a much simpler free energy where $L$ is neglected and only biquadratic coupling $P^{2} M^{2}$ is considered, the idea being to exact analytical solutions to an approximate model rather than approximate or numerical solutions to an exact model. For the sake of simplicity, the order parameters are one dimensional, and thus the model is limited to describe the behavior of $180^{\circ}$ walls only.

The biquadratic coupling term is always allowed by symmetry so it must necessarily exist in all multiferroics. Since this term is not linear, its magnitude is not limited by the Brown-Hornreich-Shtrikman upper bound ${ }^{21}$ so it can be large. This type of coupling contains implicitly the strain coupling, as electrostrictive and magnetostrictive terms all couple to the square of the order parameter, and therefore it must be important in ferroelastic multiferroics such as the perovskite ones. In spite of the simplicity of the model, the 
analytic solutions enable qualitative and even semiquantitative predictions that will be compared to the archetypal multiferroic, $\mathrm{BiFeO}_{3}$.

It will be assumed to begin with that the phase transitions can be described by a second-order quartic Landau expansion. Though the second-order transition is often valid for ferroelectric thin films, ${ }^{22}$ and has been used to describe thin films of $\mathrm{BiFeO}_{3},{ }^{23,24}$ such approximation is potentially inaccurate for bulk $\mathrm{BiFeO}_{3}$ because its ferroelectric phase transition is first order, ${ }^{5,25,26}$ which would necessarily require expansion up to at least $P^{6}$ terms. The analytical solution for the magnetoelectric domain walls in a first-order thermodynamic potential ( $P^{6}$ and $M^{6}$ terms) will therefore be presented also. Although the ferroelectric Landau coefficients have been calculated for thin films ${ }^{23,24}$ and estimated also in the Appendix of the present paper, the exact measurement of the intrinsic bulk coefficients is nevertheless still pending, and the authors very much encourage the exact measurement of all the coefficients in good quality single crystals. The domain wall magnetization estimated in this paper does come within the same order of magnitude as the remnant magnetization of $\mathrm{BiFeO}_{3}$ films, ${ }^{1}$ suggesting that it may indeed be a relevant contributor to it. A more precise treatment, using exact values of the Landau coefficients and including the symmetry and sublattice magnetization constraints neglected here, should help clarify the long-standing issue of the origin of ferromagnetism sometimes reported for this material.

\section{SECOND-ORDER APPROXIMATION $\left(P^{4}, M^{4}\right)$}

To derive the analytic solutions, we start with the LandauGinzburg potential for a magnetoelectric multiferroic with positive biquadratic coupling between $P$ and $M$, where both $P$ and $M$ are expanded to quartic terms, the minimum required to describe second-order phase transitions,

$$
G_{M P}=G_{0}+\frac{\kappa}{2}(\nabla P)^{2}+\frac{\lambda}{2}(\nabla M)^{2}+\mathfrak{I}_{M P}(P, M)
$$

with $\mathfrak{I}_{P M}$ defined as

$$
\Im_{M P}(P, M)=\frac{\alpha}{2} P^{2}+\frac{\beta}{4} P^{4}+\frac{a}{2} M^{2}+\frac{b}{4} M^{4}+\frac{\gamma}{2} P^{2} M^{2},
$$

where $\beta>0, b>0$, and $\beta b-\gamma^{2}>0$ to ensure that the nonequilibrium potential is positive definite at high value of $P$ and $M$. The coefficient multiplying the term $P^{2} M^{2}$ is an effective parameter that includes both direct coupling between $P$ and $M$ (or $E$ and $H$ ) and also indirect coupling through strain $s$, consisting of electrostriction $s P^{2}$ plus magnetostriction $s M^{2}$. Readers should note that, since biquadratic coupling is allowed by all symmetries, it will always be present, with bilinear, linear-quadratic or linear-cubic couplings being also possible for specific point groups. ${ }^{27}$

The Curie temperatures of the ferroelectric and ferromagnetic phases do not necessarily have to coincide. In several multiferroics, such as $\mathrm{BiFeO}_{3}$ or hexagonal $\mathrm{YMnO}_{3}$, the ferroelectric phase transition $\left(T_{C}\right)$ has a bigger critical temperature than the ferromagnetic or antiferromagnetic $\left(T_{\text {Neel }}\right)$ one. For $\mathrm{BiFeO}_{3}$, in particular, the $\left(P_{B} \neq 0, M_{B}=0\right)$ solutions would occur below the Curie temperature $\left(T_{C} \sim 820^{\circ} \mathrm{C}\right)$. Since $\mathrm{BiFeO}_{3}$ is an antiferromagnet below the Néel temperature $\left(T_{N} \sim 370{ }^{\circ} \mathrm{C}\right)$, we shall extend the $\left(P_{B} \neq P_{0}, M_{B}=0\right)$ solution also below the antiferromagnetic ordering temperature $\left(T<T_{N}\right)$. The case where the net magnetization is nonzero is also analyzed later.

The domain wall in $G_{M P}(x)$ is introduced in the $z-y$ plane so that $M$ and $P$ are one-dimensional functions of variable $x$ only [e.g., $M=M(x)$ and $P=P(x)$, and so is the LandauGinzburg potential $G_{M P}=G_{M P}(x)$. While equilibrium domain walls are normally flat in order to minimize elastic energy, real samples can display irregularly shaped walls ${ }^{15}$ in response to local pinning defects. A description of these is out of the scope of this paper but it is useful to point out when or whether the irregular shape of the wall affects the present analysis. A meandering domain wall can still look flat in close proximity and therefore be amenable to our model; as a rule of thumb, the present analytical model will start to lose quantitative accuracy when the local radius of curvature of the wall is comparable to the domain wall thickness. At any rate, the qualitative conclusions of the model are not dependent on the domain wall shape.

Both the domain wall energy and its polarization and magnetization profiles can be obtained by minimizing the energy difference between the system with and without domain wall in an infinite crystal. We shall construct the solutions of Eq. (1) in one dimension from the variational minimization of $G_{M P}(x)$ by employing the trial orbits $T(P, M)$ constrained to zero in the $P-M$ plane (the order-parameter space). This approach will allow us to obtain classes of analytical solutions of Eq. (1) that depend on $T(P, M)$ and $L_{M P}(P, M)$. In fact, our approach is to some extent analogous to the Rajaraman ${ }^{28}$ and Montonen ${ }^{29}$ approaches for deriving the analytical solutions of two coupled $\phi^{4}$ potentials in field theories. Indeed, starting from

$$
0=\frac{d T[P(x), M(x)]}{d x}=\frac{\partial T}{\partial P} \frac{d P}{d x}+\frac{\partial T}{\partial M} \frac{d M}{d x},
$$

together with the soliton solutions of $G_{M P}(x)$,

$$
\frac{d^{2} P(x)}{d x^{2}}=\frac{1}{\kappa} \frac{\partial \mathfrak{I}_{M P}(P, M)}{\partial P(x)}, \quad \frac{d^{2} M(x)}{d x^{2}}=\frac{1}{\lambda} \frac{\partial \mathfrak{I}_{M P}(P, M)}{\partial M(x)},
$$

we have obtained the Rajaraman condition,

$$
\left(\frac{\partial T}{\partial P}\right)^{2} \int \frac{1}{\kappa} \frac{\partial \mathfrak{I}_{M P}}{\partial P} d P=\left(\frac{\partial T}{\partial M}\right)^{2} \int \frac{1}{\lambda} \frac{\partial \mathfrak{I}_{M P}}{\partial M} d M,
$$

Eq. (4) is an integrodifferential equation in terms of $P$ and $M$. The integral has to be evaluated along $T(P, M)$ and the integration constants that are not written explicitly in Eq. (4), will vanish. The trial orbits are limited to ones having finite total action only. Periodic orbits or orbits that flow to infinity have not been considered since they have infinite total action. ${ }^{28}$ Examples of orbits with finite action, i.e., the energy has to be localized and finite as $x \rightarrow \pm \infty$, are the zeroenergy orbits that begin at one local minimum and end at another local minimum of $\mathfrak{I}_{P M}$ (e.g., another zero of $\mathfrak{I}_{P M}$ ). 
The zeros of $\mathfrak{I}_{P M}=0$, i.e., the ones that determine $P(x)$ and $M(x)$ in the middle of domains far away from the center of domain walls are

$$
\begin{gathered}
(0,0), \\
\text { I }\left( \pm P_{0}, 0\right), \\
\text { II }\left(0, \pm \sqrt{-\frac{a}{b}}\right), \\
\text { III }\left( \pm \sqrt{\frac{a \gamma-\alpha b}{\beta b-\gamma^{2}}}, \pm \sqrt{\frac{\alpha \gamma-a \beta}{\beta b-\gamma^{2}}}\right),
\end{gathered}
$$

where $P_{0}=\sqrt{-\frac{\alpha}{\beta}}$. Note that the minima of $\mathfrak{I}_{P M}$, which are its zeros [see. Eq. (5)], minimize also the Landau-Ginzburg potential. $^{28}$

Readers should note that the model (1) describes secondorder transitions (i.e., ones associated with continuous changes in order parameters $P$ and $M$ at phase-transition temperature) that the system may undergo with temperature from the high temperature (high symmetry) toward the lowtemperature (low-symmetry) phases. ${ }^{30}$

Let us look at $\left( \pm P_{0}, 0\right)$, which corresponds to the lowsymmetry phase I characterized by a ferroelectric moment only (i.e., a ferroelectric domain wall). The stability condition $\partial^{2} \Im_{P M} / \partial P^{2}>0$ in this phase requires that $\gamma>-a / P_{0}^{2}$ to allow $\left(-P_{0}, 0\right)$ and $\left(+P_{0}, 0\right)$ to be local minima of $\mathfrak{I}_{M P}$. This condition also provides that the other zeros of $\mathfrak{I}_{M P}=0$ (i.e., II and III) do no correspond to any local minima of $\mathfrak{I}_{M P}$. As noted above, $P$ and $M$ are constrained to go from one local minimum $\left(-P_{0}, 0\right)$ to another minimum $\left(+P_{0}, 0\right)$. A zero-energy orbit for $\left( \pm P_{0}, 0\right)$ would be, for example, 0 $\equiv T(P, M)=f\left(P^{2}-P_{0}^{2}\right)+M^{2}$, which together with Eqs. (3) and (4), determines the $P$ (kink) $-M$ (breather) domain wall solution that minimizes both $\mathfrak{I}_{M P}$ and $G_{M P}$. The kink-breather solution is a topological soliton (topological number different from zero) derived first in the field theory. ${ }^{28,29}$ Only a limited number of zero-energy orbits are found to minimize Eq. (1) and satisfy Eq. (4) simultaneously. Solutions constrained on orbits as $T(P, M)=f\left(P^{2}-P_{0}^{2}\right)+M^{r}$, where $r>2$, are excluded, as they do not satisfy Eq. (4) or minimize Eq. (1). Readers should note that all possible domain wall profiles minimizing Eq. (1) depend on the degree of complexity of $T(P, M)$, and therefore cannot be derived explicitly from Eq. (4). Kink-breather domain wall profiles satisfying Eq. (4) have been systematically calculated from Eq. (1) for all tested zero-energy orbits that start at $\left(-P_{0}, 0\right)$ and end at $\left(+P_{0}, 0\right)$.

In the ferroelectric phase I, very different scenarios take place depending on the relative signs and magnitudes of the coefficient $a$ that multiplies $M^{2}$ and the magnetoelectric coupling coefficient $\gamma$. It is easy to visualize this by considering only the magnetic and magnetoelectric parts of the free energy, $\mathfrak{I}_{M}(M)=\frac{a}{2} M^{2}+\frac{b}{4} M^{4}+\frac{\gamma}{2} P^{2} M^{2}$. The terms multiplying $M^{2}$ may be regrouped as

$$
\mathfrak{I}_{M}(M)=\left(\frac{a}{2}+\frac{\gamma}{2} P^{2}\right) M^{2}+\frac{b}{4} M^{4} .
$$

From this regrouping, it emerges that the effect of the magnetoelectric coupling is a renormalization of the lowestorder magnetic coefficient, and hence of the magnetic transition. Assuming a positive sign for $\gamma$, and given that $P \neq 0$ in the ferroelectric state, the magnetoelectric coupling makes the magnetic coefficient "more positive." If $a>0$ (i.e., if $T$ $>T_{\text {Neel }}$ ), this is of no consequence whatsoever. But, if $a$ $<0\left(T<T_{\text {Neel }}\right)$, then something very interesting may happen. Specifically, if $a<0$ (i.e., if the material is below $T_{\text {Neel }}$ ) but the absolute value of the coefficients is such that $\frac{a}{2}+\frac{\gamma}{2} P^{2}$ $\geq 0$, then the material will still display no magnetism. Except, of course, where the polarization is suppressed, i.e., inside the domain wall. In the middle of the domain wall, the polarization is zero and hence the magnetoelectric term, $\frac{\gamma}{2} P^{2}$, is cancelled so that magnetism can emerge. The analytic expressions for $P(x)$ and $M(x)$ in this scenario, calculated from the Landau-Ginzburg potential Eq. (1), are

$$
P(x)=P_{0} \tanh \left(\frac{x}{\delta_{M P}}\right), \quad M(x)= \pm M_{0} \operatorname{sech}\left(\frac{x}{\delta_{M P}}\right),
$$

where $M_{0}$ is the magnetization in the center of the domain wall [see Fig. 1(b)] and $P_{0}$ is the polarization in the center of the domains [see Fig. 1(a)],

$$
P_{0} \equiv \sqrt{-\frac{\alpha}{\beta}}, \quad M_{0} \equiv \sqrt{\frac{\gamma \alpha-2 a \beta}{\beta b}},
$$

where $a<0$ (ferroelectric state). $P(x)$ and $M(x)$ are constrained on an ellipse $M^{2} / M_{0}^{2}+P^{2} / P_{0}^{2}=1$ in the $P-M$ plane [see Fig. 1(c)]. A similar relationship between the order parameters has been obtained in the spherical model and its relaxor relative, the spherical random-bond, random-field model. ${ }^{31}$ The kink-breather domain wall profile has the homotopic invariant, $I(P, M) \equiv \int d x(P \times \partial M / \partial x)$ equals in magnitude to the orbit area. As $I(P,+M)=-I(P,-M)$ therefore, the kink-breather domain wall profile would possess total magnetoelectric chirality different from zero depending on which branch of the ellipse $M$ travels along [Fig. 1(c)]. This result complements Lajzerowicz's ${ }^{9}$ who predicted a similar effect in ferroelastic domain walls. These authors also showed that the existence of coupling between two ferroic order parameters may lead to chirality, which accounts for the observation that the ferroelectric domain walls of $\mathrm{BiFeO}_{3}$ are Heisenberg-type rather than Ising-type, that is, the ferroelectric polarization rotates across the wall. ${ }^{32}$ Incidentally, the existence of chirality inside $180^{\circ}$ walls has also been proposed for nonmagnetoelectric ferroelectrics such as $\mathrm{BaTiO}_{3} ;{ }^{33}$ although these are not magnetic they are still multiferroic as they are ferroelectric and ferroelastic, hence the chirality predictions of Lajzerowicz ${ }^{9}$ may be applied to it.

Equations (7) and (8) show that a net magnetization can appear in the domain walls due to the magnetoelectric coupling and the gradient terms, even when the domains themselves have no net magnetization. Similarly, Goltsev and Lottermoser ${ }^{11,12}$ have reported that antiferromagnetic multiferroics, such as $\mathrm{YMnO}_{3}$ and $\mathrm{HoMnO}_{3}$, can also reveal intrinsic net magnetization in the center of the domain walls due to magnetoelectric coupling. Our result agrees with concepts discussed in Refs. 9-12 and extends them to the ana- 
(a)

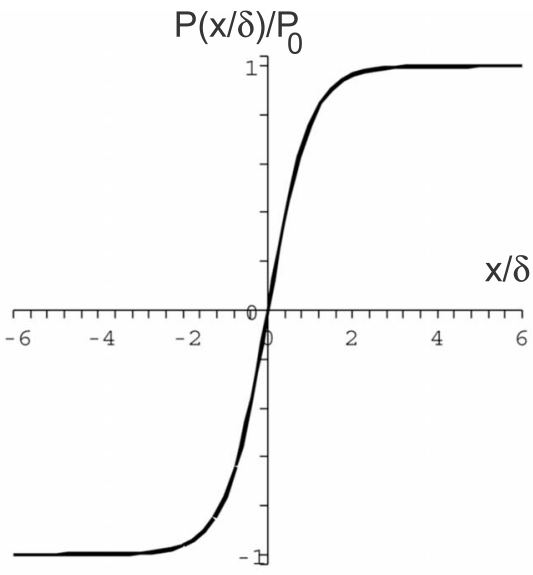

(c)

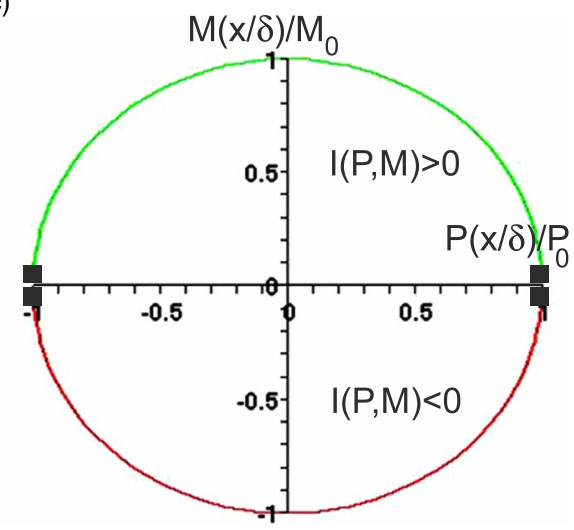

(b)

$\mathrm{M}(\mathrm{x} / \delta) / \mathrm{M}_{0}$

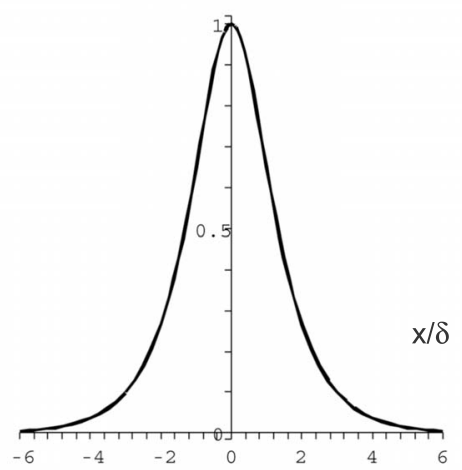

(d)

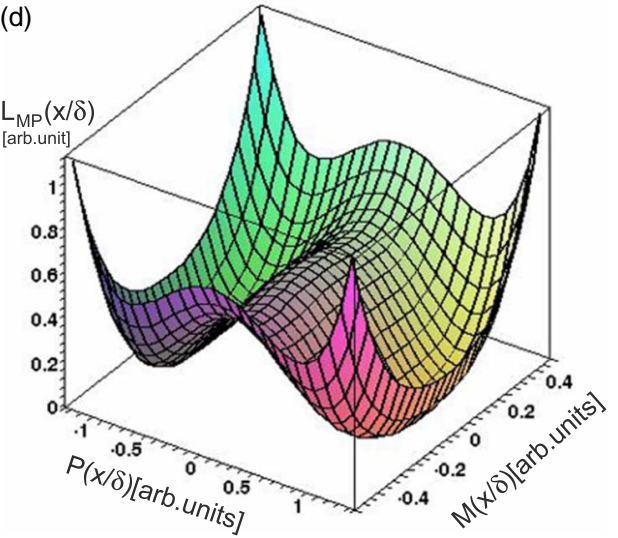

FIG. 1. (Color online) Polarization-magnetization domain wall profile when domains are not ferromagnetic: (a) $P \equiv 0$ in the center of domain walls but $P \neq 0$ in the domains; (b) $M \neq 0$ in the center of domain walls but $M \equiv 0$ in the domains; (c) $M(x)$ and $P(x)$ are constrained on an ellipse; (d) $\mathfrak{I}_{M P}(x)$ shows two minima at $P= \pm P_{0}$. The system will move from one of those minima to other through a maximum value at 0 . This will gives an increase in $M$ in the center of domain walls. The filled squares in (c) represent the minima of $\mathfrak{I}_{M P}(x)$.

lytical solutions of spatial variation in magnetization and polarization to the entire width of the domain walls as functions of domain wall correlation length. The domain wall correlation length is given by

$$
\delta_{M P} \equiv 2^{1 / 2} \sqrt{\left(\frac{\beta b \kappa}{2 \gamma \beta a-\alpha \gamma^{2}-\alpha \beta b}\right)}
$$

and the Landau coefficient $\kappa>0, \lambda>0, \alpha<0, \beta>0, a<0$, $b>0$, and $\gamma>0$. Equation (9) shows that the ferroelectric domain wall thickness $\left(2 \times \delta_{M P}\right)$ in magnetoelectrics will always be wider than in pure ferroelectrics, $2 \times \delta_{P}$, where $\delta_{P}$ $=2^{1 / 2}(-\kappa / \alpha)^{1 / 2}$. These results are only fulfilled at condition $\gamma>a \beta / \alpha$, which states explicitly that at the low-temperature phase $\left( \pm P_{0}, 0\right)$ the coupling has to be strong enough in the quartic Landau expansion to allow the magnetization to come into existence at the middle of domain walls. Yet another scenario takes place when $a<0$ and $\frac{a}{2}+\frac{\gamma}{2} P^{2} \leq 0$. In this case, the material has net magnetism both inside and outside the domains but, crucially, as shown by Eq. (6), the coefficient multiplying the $M^{2}$ term is more negative (and hence the magnetism bigger) inside the domain walls than outside, due to the suppression of the magnetoelectric coupling. Hence, an increase over the bulk magnetism may be ex- pected at the walls also when the material is already in a magnetic state. This situation may apply to bismuth ferrite thin films, which are known to have a canting moment below $T_{\text {Neel }}$. Because $\mathrm{BiFeO}_{3}$ has a first-order phase transition, though, this situation is best analyzed using a LandauGinzburg-Devonshire potential expansion up to sixth-order terms.

\section{SIXTH-ORDER EXPANSION}

Strictly speaking, the model (1) should not be applicable to bismuth ferrite, as the ferroelectric transition in this material is found experimentally to be first order, ${ }^{5}$ which requires the ferroelectric order parameter to be expanded up to order $P^{6}$-although the approximation to $P^{4}$ terms has nevertheless been used to describe strain effects in thin films of this material. ${ }^{23}$ Toledano, ${ }^{34}$ Gufan, ${ }^{35}$ and Holakovsky ${ }^{36}$ have shown that the model depicted by Eq. (1) is unsuitable for negative interactions between the order parameters $(\gamma<$ $-\sqrt{\beta b}$ ), which may limit the model's applicability in the description of experimental results in the $\left( \pm P_{0}, 0\right)$ phase. By including $P^{6}$ and $M^{6}$ terms in Eq. (1) we may show that the kink-breather solution [Eq. (7)] is again a solution of the 
amended potential and overcomes the constraint on $\gamma$ to be large in the $\left(P_{B} \neq 0, M_{B}=0\right)$ phase in order to induce domain wall magnetization in the quartic Landau model. Thus, we consider a potential expanded up to $P^{6}$ and $M^{6}$ terms,

$$
G_{M P}=G_{0}+\frac{\kappa}{2}(\nabla P)^{2}+\frac{\lambda}{2}(\nabla M)^{2}+\mathfrak{I}_{M P}(P, M)
$$

with a potential $\mathfrak{I}_{P M}$ defined as

$$
\begin{aligned}
\Im_{M P}(P, M)= & G_{0}+\frac{\kappa}{2}(\nabla P)^{2}+\frac{\lambda}{2}(\nabla M)^{2}+\frac{\alpha}{2} P^{2}+\frac{\beta}{4} P^{4}+\frac{\eta}{6} P^{6} \\
& +\frac{a}{2} M^{2}+\frac{b}{4} M^{4}+\frac{n}{6} M^{6}+\frac{\gamma}{2} P^{2} M^{2}
\end{aligned}
$$

$n>0$ and $\eta>0$ ensure that $G_{M P}$ is positive definite at high value of $P$ and $M$. Some zeros of $\mathfrak{I}_{P M}$ are

$$
\left( \pm P_{0}, 0\right)
$$

$$
\left(0, \pm \sqrt{\frac{-b+\sqrt{b^{2}-4 a n}}{2 n}}\right)
$$

where $P_{0}=\sqrt{\frac{-\beta+\sqrt{\beta^{2}-4 \alpha \eta}}{2 \eta}}$.

In the low-symmetry $\left(P_{B} \neq 0, M_{B} \neq 0,\right)$ phase, equivalent to III in Eq. (5), $M_{B}$ and $P_{B}$ are solutions of the following polynomial equations to the eighth power

$$
M_{B}=\sqrt{\eta n^{2} x^{8}+2 b \eta n x^{6}+\left(-\beta \gamma n+\eta b^{2}-2 \eta n a\right) x^{4}+\left(-\beta \gamma b-2 \eta a b+\gamma^{3}\right) x^{2}-\alpha \gamma^{2}+\beta \gamma b+\eta a^{2}},
$$

$$
P_{B}=\sqrt{\frac{a-b M_{B}^{2}-d M_{B}^{4}}{\gamma}} .
$$

\section{A. Ferroelectric, nonmagnetic domains, $P \neq 0, M=0$}

In Eq. (11), we look at the case $\alpha<0$ and $a>0$ in the intermediate phase $T_{N e e l}<T<T_{C}$, which states explicitly that the domains are in the ferroelectric state of polarization $P_{0}$ above the magnetic ordering $\left(M_{B}=0\right)$ temperature. Though $\gamma$ is normally positive, it can be small or even slightly negative in Eq. (10). We again consider a zero-energy orbit 0 $\equiv T(P, M)=f\left(P^{2}-P_{0}^{2}\right)+M^{2}$ for $\left(-P_{0}, 0\right)$ and $\left(+P_{0}, 0\right)$ minima to calculate the domain wall profiles that minimize Eq. (10) and satisfy Eq. (4) simultaneously. The analytic expressions for $P(x)$ and $M(x)$ across the domain walls in the stability field of low-symmetry $\left(P_{B}= \pm P_{0}, M_{B}=0\right)$ phase are

$$
\begin{gathered}
M(x) \equiv \frac{\sqrt{2 c_{1}}}{\sqrt{\sqrt{c_{2}^{2}-3 c_{1} c_{3}} \cosh \left(2 \sqrt{c_{1}} x\right)+c_{2}}}, \\
c_{1}=\frac{a+\gamma P_{0}^{2}}{\lambda}, \\
c_{2}=\frac{\left(16 n P_{0}^{8} \eta^{2}+24 n P_{0}^{6} \eta \beta+9 n P_{0}^{4} \beta^{2}+27 \gamma^{3} P_{0}^{2}+27 \gamma^{2} a\right)}{6 \gamma P_{0}^{2} \lambda\left(4 \eta P_{0}^{2}+3 \beta\right)}, \\
c_{3}=\frac{n}{\lambda},
\end{gathered}
$$

and

$$
P(x) \equiv \sqrt{P_{0}^{2}-\frac{3 \gamma}{4 \eta P_{0}^{2}+3 \beta} M(x)^{2}},
$$

where $\alpha<0, a>0, \gamma \geq 0$, and

$$
\delta_{M P} \equiv \frac{1}{2 \sqrt{c_{1}}}=\sqrt{\frac{\lambda}{4\left(a+\gamma P_{0}^{2}\right)}} .
$$

Studying Eqs. (12) and (13), one observes that they have standard forms of bell-shaped $M(x)$ and kink-shaped $P(x)$ as those in Fig. 1. $M(x)$ and $P(x)$ are again constrained on an ellipse. The limit $\gamma \rightarrow 0$ forces $P(x) \rightarrow P_{0}$ and $M(x) \rightarrow 0$ in Eqs. (11) and (12), a result that is intuitively expected to be correct when $M(x)$ and $P(x)$ are decoupled in the LandauGinzburg potential. A large $\gamma \gg 0$ would induce a dramatic increase in $M(x)$ in the middle of domain walls when $P(x)$ inverts through zero (see Fig. 1), while, $\gamma \rightarrow 0$ will not induce any magnetic moments within domain walls with inversion of $P(x)$ at zero, and thus the material will remain nonmagnetic both within the walls and, of course, within the domains.

Building on Eqs. (12)-(14), and using the Landau coefficients estimated in Appendix, we calculate $\delta_{M P}=6$ $\times 10^{-9} \mathrm{~m}, M_{0}=1.3 \times 10^{5} \mathrm{~A} / \mathrm{m}=130 \mathrm{emu} / \mathrm{cm}^{3}$ for $\mathrm{BiFeO}_{3}$ [see Fig. 2(b)]. This magnetization inside the domain wall is very large, as it represents nearly 1 bohr magnetron per unit cell. It is also noteworthy that the expected ferroelectric domain wall thickness of $\mathrm{BiFeO}_{3}$ is considerably thicker than that of "pure" ferroelectrics, and closer to that of magnetic domain walls. Specifically, the domain wall thickness $D$ $=2 \delta_{M P}=12 \mathrm{~nm}$ is comparable to that of pure ferromagnets 

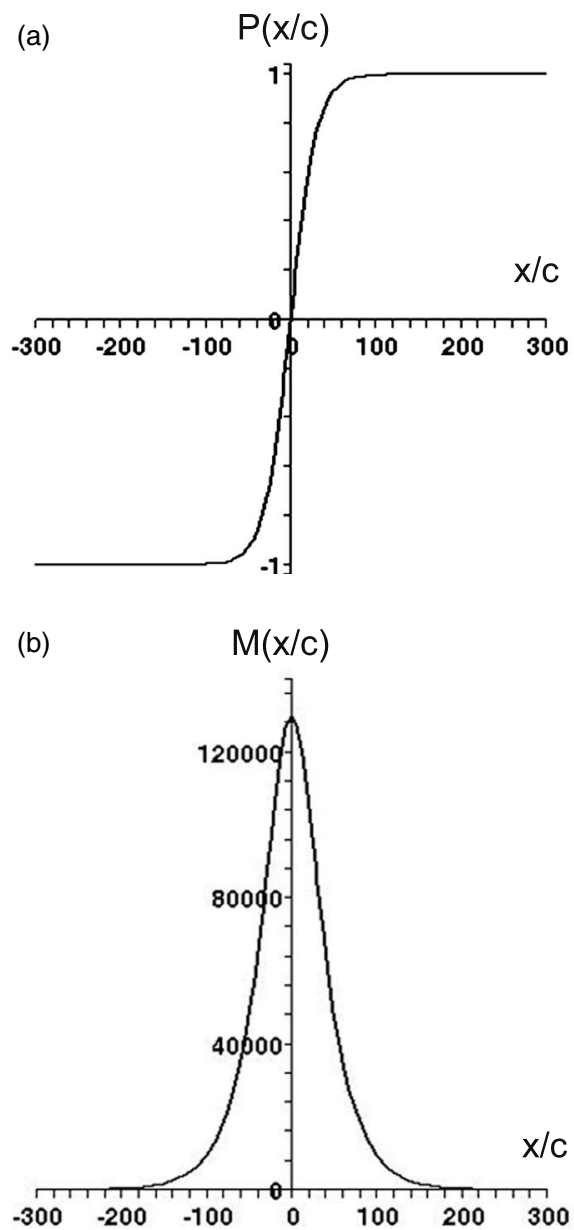

FIG. 2. (a) and (b) Polarization-magnetization domain wall profile in $\mathrm{BeFeO}_{3} ; M(x / c)$ and $P(x / c)$ are constrained on an ellipse [Eq. (12)]. The system will move from $P_{0}=-1$ to $P_{0}=+1$, giving an increase in $M$ in the center of domain walls. The Landau coefficients are given in Appendix. The $x$ axis is normalized to $x / c$, where $c=4 \AA$ is the lattice constant.

with high anisotropy, such as cobalt. This result had been anticipated on the basis of the big domain periodicity observed in $\mathrm{BiFeO}_{3}$ thin films ${ }^{15}$ and the known proportionality between domain size and domain wall thickness. ${ }^{16}$

The remnant magnetization of Wang's samples ${ }^{1}$ for both the in-plane and the out-of-plane loops measured on 70-nmthick $\mathrm{BiFeO}_{3}$ film by vibrating sample magnetometry is $M_{\text {remnant }} \sim 10-15 \mathrm{emu} / \mathrm{cm}^{3}$, with the reported saturation magnetization being considerably bigger, around $150 \mathrm{emu} / \mathrm{cm}^{3}$ (i.e., almost $1 \mu_{B}$ per unit cell), although more recent measurements give a lower value around $60 \mathrm{emu} / \mathrm{cm}^{3} .{ }^{37}$ Our intention is to quantify whether this experimental $M_{\text {remnant }}$ may be caused by the domain wall magnetization. For this, we approximate the average contribution of domain wall magnetization to the macroscopic magnetization of the film as $M_{D W} \equiv M_{0} D / w=M_{0} \times 2 \delta_{M P} / w$, where the ratio $D / w$ measures the volume fraction of domain walls in the film. Typical values for the stripe domain widths in the thin films are in the region $w \sim 100 \times 10^{-9}-400 \times 10^{-9} \mathrm{~m}$ (Ref. 38) with fractal domains being considerably smaller ${ }^{15}$ so that their domain wall contribution may be larger. Using
$\delta_{M P}=6 \times 10^{-9} \mathrm{~m}, w=200 \mathrm{~nm}$, and $M_{0}=1.3 \times 10^{5} \mathrm{~A} / \mathrm{m}$ in the center of the walls this leads to a net macroscopic magnetization of $M_{D W} \sim 7800 \mathrm{~A} / \mathrm{m} \sim 8 \mathrm{emu} / \mathrm{cm}^{3}$, which is reasonably close to the experimental remnant magnetization given the crudeness of some of our approximations. This, of course neglects the already existing canting moment of $\mathrm{BiFeO}_{3}$, which is also on the order of $6-8 \mathrm{emu} / \mathrm{cm}^{3}$. If we simply add the two, we obtain a net magnetization on the order of $14-16 \mathrm{emu} / \mathrm{cm}^{3}$, which is close to the value experimentally measured.

Our results therefore show that domain walls could, in principle, account for a significant fraction of the enhancement in remnant magnetization in $\mathrm{BiFeO}_{3}$ thin films, particularly for films with fractal walls, which have smaller domains and thus a larger perimeter-to-area ratios (i.e., bigger ratios of wall to domain). The domain wall magnetization, on the other hand, is still too small to explain the large measured saturation magnetization, although we emphasize that our calculations are done only for zero external field and do not really attempt to address what happens at saturation fields. Of course, our solution does not exclude further contributions from any of the other explanations already put forward in the literature such as vacancy-induced $\mathrm{Fe}^{2+}$ oxidation state, ${ }^{6}$ parasitic ferromagnetic phases, ${ }^{7}$ or localized "hotspots" of magnetite generated upon electric field cycling. ${ }^{8}$

It is also worth mentioning that domain walls are known to attract charge carriers and oxygen vacancies. ${ }^{39-41}$ These could further enhance the local magnetization at the domain wall beyond the value calculated here. Thus, domain walls may contribute to the magnetization both intrinsically, as calculated above, or extrinsically via concentration of oxygen vacancies in their interior. This latter mechanism would also provide an explanation for the fact that, while $\mathrm{BiFeO}_{3}$ magnetization is known to be linked to the $\mathrm{O}$ pressure during fabrication, the level of $\mathrm{O}$ vacancies required to explain the magnetization is too high to be compatible with the pristine structure observed by $\mathrm{x}$-ray diffraction; if $\mathrm{O}$ vacancies were not in the domains but localized inside the narrow domain walls, they would avoid being observed by macroscopically averaged techniques such as X-ray diffraction while still contributing to strong localized magnetization.

On the other hand, other groups have not observed any enhancement in remnant magnetization ${ }^{7}$ in spite of the fact that their films clearly possess domain walls. ${ }^{15}$ Two explanations may be invoked here. First, the type of domain wall is important. Our analysis is for $180^{\circ}$ domain walls, i.e., those where the polarization is completely reversed. $\mathrm{BiFeO}_{3}$, however, is known to have also ferroelectric/ferroelastic domain walls where the polarization rotates by approximately $\sim 109^{\circ}$ (separating domains where only two of the Cartesian components of the 111-oriented polarization are reversed) and $\sim 71^{\circ}$ (those where only one component is reversed). Naively one may expect the magnetization in these walls to be a fraction of that in the $180^{\circ}$ wall; if the magnetization is only enhanced for the fraction of polarization that is reversed, then the $109^{\circ}$ walls will have smaller magnetization than the 180 walls but bigger than $71^{\circ}$ walls. In this respect, it is noteworthy that Ramesh et al. have noticed that films with only $71^{\circ}$ walls have no appreciable enhancement of 

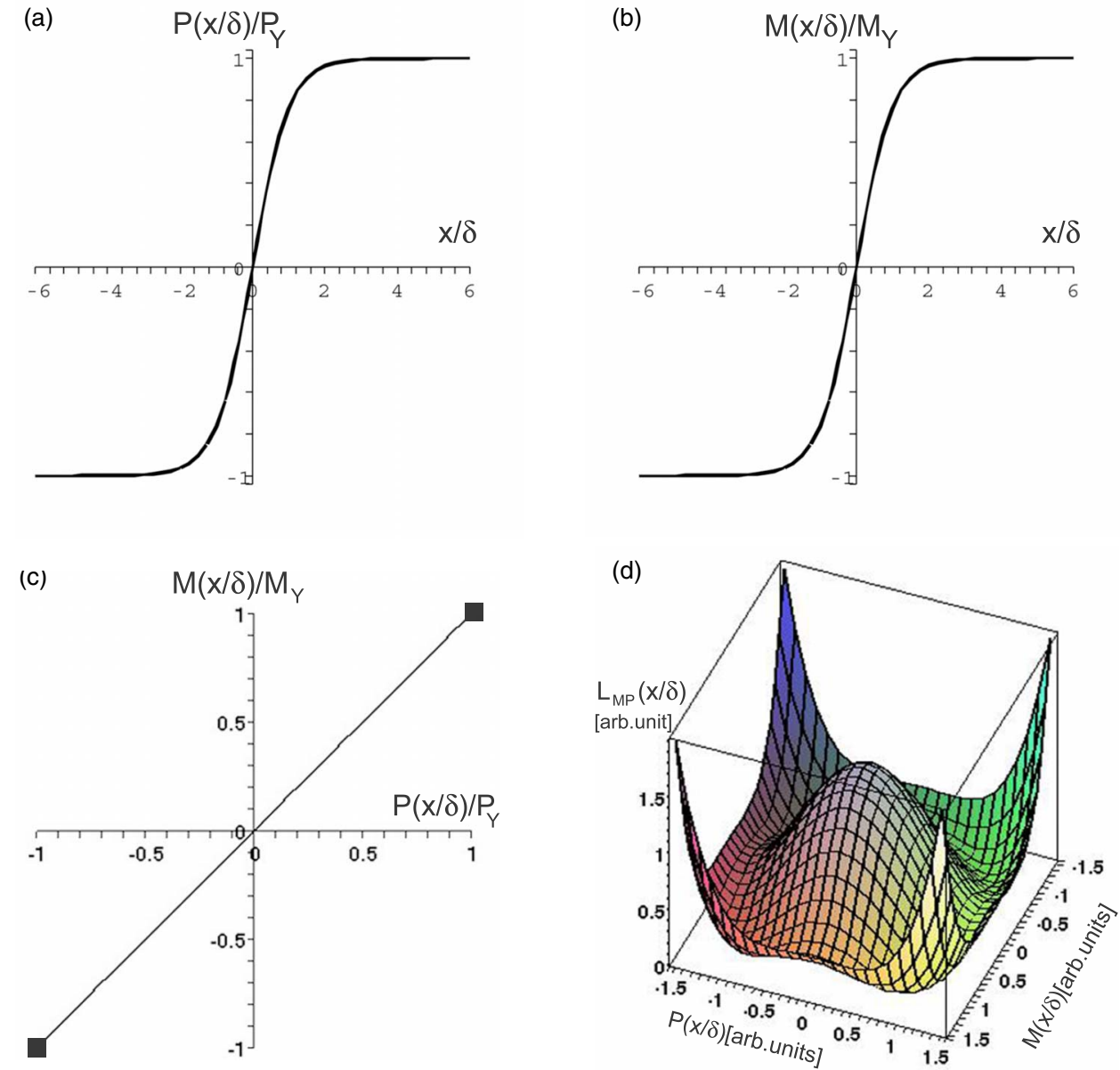

FIG. 3. (Color online) Polarization-magnetization domain wall profiles in the stability field of the low-symmetry phase with $M_{B}$ and $P_{B}$. The underlying equations are Eqs. (15) and (16). (a) and (b) Both $M$ and $P$ are inverted; (c) $M(x)$ and $P(x)$ are constrained on a line, (d) $\mathfrak{I}_{M P}(x)$ shows four minima at $\left( \pm P_{B}, \pm M_{B}\right)$. The system will move from one of these minima (i.e., $\left.-P_{B},-M_{B}\right)$ toward the opposite one (e.g., $\left.+P_{B},+M_{B}\right)$ through a maximum at zero. This forces $M(0)=0$ and $P(0)=0$. The filled squares in (c) represent some of the minima of $\mathfrak{I}_{M P}(x)$.

magnetization, in contrast with those with $109^{\circ}$ walls which do. ${ }^{37}$ Since different substrate orientations, deposition rates and electrode configurations favor different type of domains, it is conceivable that the samples from different laboratories might have different predominance of one type or another of domain wall, thereby accounting for the different magnetic behavior. A detailed study of the correlation between domain type and magnetization is needed in order to clarify this point.

\section{B. Ferroelectric and magnetic domains}

While bulk $\mathrm{BiFeO}_{3}$ is an antiferromagnet with zero net magnetization on account of the cycloidal spin rotation, in thin films the spin cycloid is destroyed. This leads to weakly canted spin configuration with weak ferromagnetism (ferrimagnetism) which can be further enhanced by impurities or doping. Accordingly, it is perhaps inappropriate to treat $\mathrm{BiFeO}_{3}$ thin films as having strictly zero net magnetization in zero field, as done above, and instead one should treat it as a material which is both ferroelectric and (weakly) ferromagnetic; in mathematical terms of the Landau theory, one where both $\alpha$ and $a$ (the first-order coefficients of the polarization and the magnetization) are negative. Therefore, $M$ would be nonzero in the domains, $M_{B} \neq 0$.

The solutions for ferroelectric-ferromagnetic domain walls are derived analytically from Eqs. (1) and (2) by constraining them on a zero-energy orbits that begin and end at

$$
P_{B}= \pm \sqrt{\frac{a \gamma-\alpha b}{\beta b-\gamma^{2}}}, \quad M_{B}= \pm \sqrt{\frac{\alpha \gamma-a \beta}{\beta b-\gamma^{2}}},
$$

$\alpha<0$ and $a<0$ in Eq. (15). Those are solutions of the lowsymmetry phase characterized by both ferroelectric and ferromagnetic moments. The stability conditions $\partial^{2} \mathfrak{I}_{P M} / \partial P^{2}$ $>0, \partial^{2} \mathfrak{I}_{P M} / \partial M^{2}>0$, and $\partial^{2} \mathfrak{I}_{P M} / \partial M / \partial P>0$ in this phase provide that $\gamma<a \beta / \alpha$ or $\gamma<\alpha b / a$, meaning that $\gamma$ has to be small enough to allow $\left(-P_{B},-M_{B},\right),\left(-P_{B},+M_{B},\right),\left(+P_{B}\right.$, $\left.-M_{B},\right)$, and $\left(+P_{B},+M_{B}\right)$ to be local minima of $\mathfrak{I}_{M P}$ [see Fig. $3(\mathrm{~d})]$. This condition also provides that the other zeros of $\mathfrak{I}_{M P}=0$, i.e., $\left(-P_{0}, 0\right)$ and $\left(+P_{0}, 0\right)$, do no correspond to any local minima of $\mathfrak{I}_{M P}$. The potential $\mathfrak{I}_{M P}$ will have four zeros [see Fig. 3(d)] that define all possible orientations (up and down direction) of $P$ and $M$ moments in domains. This contrasts the potential at Fig. 1(d) which has two zeros with two possible orientation of $P$ (up and down) in domains. 


\section{Walls that invert $P$ and $M$ simultaneously}

The variational minimization of Eq. (1) constrained on the zero-energy orbit $0 \equiv T(P, M)=f\left(P^{2}-P_{B}^{2}\right)+\Re\left(M^{2}-M_{B}^{2}\right)$, together with Eqs. (3) and (4), determines the $P$ (kink)- $M$ (kink) domain wall solution (i.e., the wall where both polarization and magnetization get inverted). Similarly to the kink-breather solution discussed above (see Fig. 1 and 2), the kink-kink solution is also a topological soliton solution studied first in the field theory. ${ }^{28,29}$ An interesting, and so far unresolved, question is whether the domain wall thickness is the same for the two different order parameters $(P$ and $M)$. There are two extreme scenarios. In the case of weak coupling, analyzed by Goltsev et al., ${ }^{11}$ the two correlation lengths for the two order parameters are almost independent so that the ME coupling merely introduces a small perturbation that does not modify very substantially the purely magnetic and purely ferroelectric walls. As a result, the magnetic thickness of the domain wall remains big while the ferroelectric thickness of the domain wall remains narrow. At the other end of the scale, in strongly coupled systems where the two order parameters share the same transition temperature, the two correlation lengths may be the same: an example of this are the ferroelastic/ferroelectric $90^{\circ}$ walls in a typical perovskite ferroelectric such as $\mathrm{BaTiO}_{3}$, where the spontaneous strain is completely linked to the ferroelectric polarization.

When there is a unique domain wall thickness, the kinkkink solution is just a double tanh function for the two order parameters,

$$
\begin{gathered}
P(x)=P_{Y} \tanh \left(x / \delta_{Y}\right), \\
M(x)= \pm M_{Y} \tanh \left(x / \delta_{Y}\right) .
\end{gathered}
$$

For simplicity we limit ourselves here to magnetoelectric domain walls in which both order parameters have the same (coupled) coherence length $\delta_{Y}$. The limiting scenarios can be as thin as the purely ferroelectric walls,

$$
\begin{gathered}
P_{Y} \equiv P_{B}, \\
M_{Y} \equiv M_{B}, \\
\delta_{Y} \equiv 2^{1 / 2} \sqrt{\frac{\kappa}{\gamma M_{B}^{2}+\beta P_{B}^{2}}}=2^{1 / 2} \sqrt{-\frac{\kappa}{\alpha}},
\end{gathered}
$$

or by contrast be as thick as the purely magnetic walls,

$$
\begin{gathered}
P_{Y} \equiv \sqrt{\frac{a}{\lambda} \frac{(\lambda \gamma-\kappa b)}{\left(\beta b-\gamma^{2}\right)},} \\
M_{Y} \equiv \sqrt{-\frac{a}{\lambda} \frac{(\lambda \beta-\kappa \gamma)}{\left(\beta b-\gamma^{2}\right)}}, \\
\delta_{Y} \equiv 2^{1 / 2} \sqrt{-\frac{\lambda}{a}},
\end{gathered}
$$

$\alpha<0$ and $a<0$ in Eq. (16). The domain walls [Eq. (16)] do not contribute to the remnant magnetization as $P(0)=0$,
$M(0)=0$. Meanwhile, $P(x)$ and $M(x)$ are constrained on a line [see Fig. 3(c)] with equation

$$
M(x)= \pm \frac{M_{Y}}{P_{Y}} P(x) .
$$

The linear proportionality in Eq. (17) does not emerge from a linear coupling term in the free energy. Bulk linear coupling between $P$ and $M$ can be found in antiferromagnets where the symmetry allows a Dzialoshinskii-Moriya-type interaction between the polarization and a spin canting moment $(M) .{ }^{18-20} \mathrm{In} \mathrm{BiFeO}_{3}$ such coupling exists but is averaged out by the cycloid; in samples where the cycloid is cancelled (e.g., very thin films ${ }^{1}$ or doped samples ${ }^{42}$ ), linear coupling may be recovered. ${ }^{43}$

\section{Walls that invert polarization but not magnetization}

Importantly for our purpose, $P(x)$ and $M(x)$ across the domain walls in the stability field of low-symmetry $\left(P_{B}, M_{B}\right)$ phase can also obey the following relations (see Fig. 4),

$$
\begin{gathered}
P(x)=P_{C} \tanh \left(x / \delta_{C}\right), \\
M(x)=M_{C} \sqrt{2-\tanh ^{2}\left(x / \delta_{C}\right)},
\end{gathered}
$$

where

$$
\begin{gathered}
P_{C}= \pm \frac{\sqrt{2}}{2} \sqrt{\frac{2 \beta b P_{B}^{2}+\gamma b M_{B}^{2}-\gamma^{2} P_{B}^{2}}{\beta b}}, \\
M_{C}= \pm \frac{\sqrt{2}}{2} \sqrt{\frac{\gamma P_{B}^{2}+b M_{B}^{2}}{b}},
\end{gathered}
$$

and

$$
\delta_{C}=\frac{2}{P_{B}} \sqrt{\frac{\kappa b}{\left(b \beta-\gamma^{2}\right)}}
$$

with $\alpha<0, a<0$.

This type of domain wall corresponds to an inversion of ferroelectric polarization without inversion of magnetic moment. Such walls would be thicker than one in pure ferroelectrics and, by contrast to the scenario depicted by Eqs. (16) and (17), here the domain walls would contribute to an enhanced remnant magnetization as $P(0)=0, M(0)=\sqrt{-a / b}$.

$P(x)$ and $M(x)$ are constrained on

$$
\frac{M(x)^{2}}{M_{C}^{2}}+\frac{P(x)^{2}}{P_{C}^{2}}=2
$$

showing that domain wall profile would also possess magnetoelectric chirality. The profile of $\mathfrak{I}_{M P}$ is similar to that drawn in Fig. 1(d) with two local minima and therefore, will not be plotted in Fig. 4. Figure 1(d) reveals that the system moves from one of $\mathfrak{I}_{M P}$ minima toward another minimum without reverting $M(x)$ [i.e., bell-shaped $M(x)$ in Fig. 4(b)]. However, $P(x)$ will invert at zero giving $P(0)=0$ [i.e., kinkshaped $P(x)$ in Fig. 4(a)]. Furthermore, from Eq. (18), $M$ $=\sqrt{2} M_{C}$ in the center of domain walls while $M=M_{C}$ in the domains [see Fig. 4(b)] so that the domain wall magnetization is only fractionally enhanced with respect to the total 

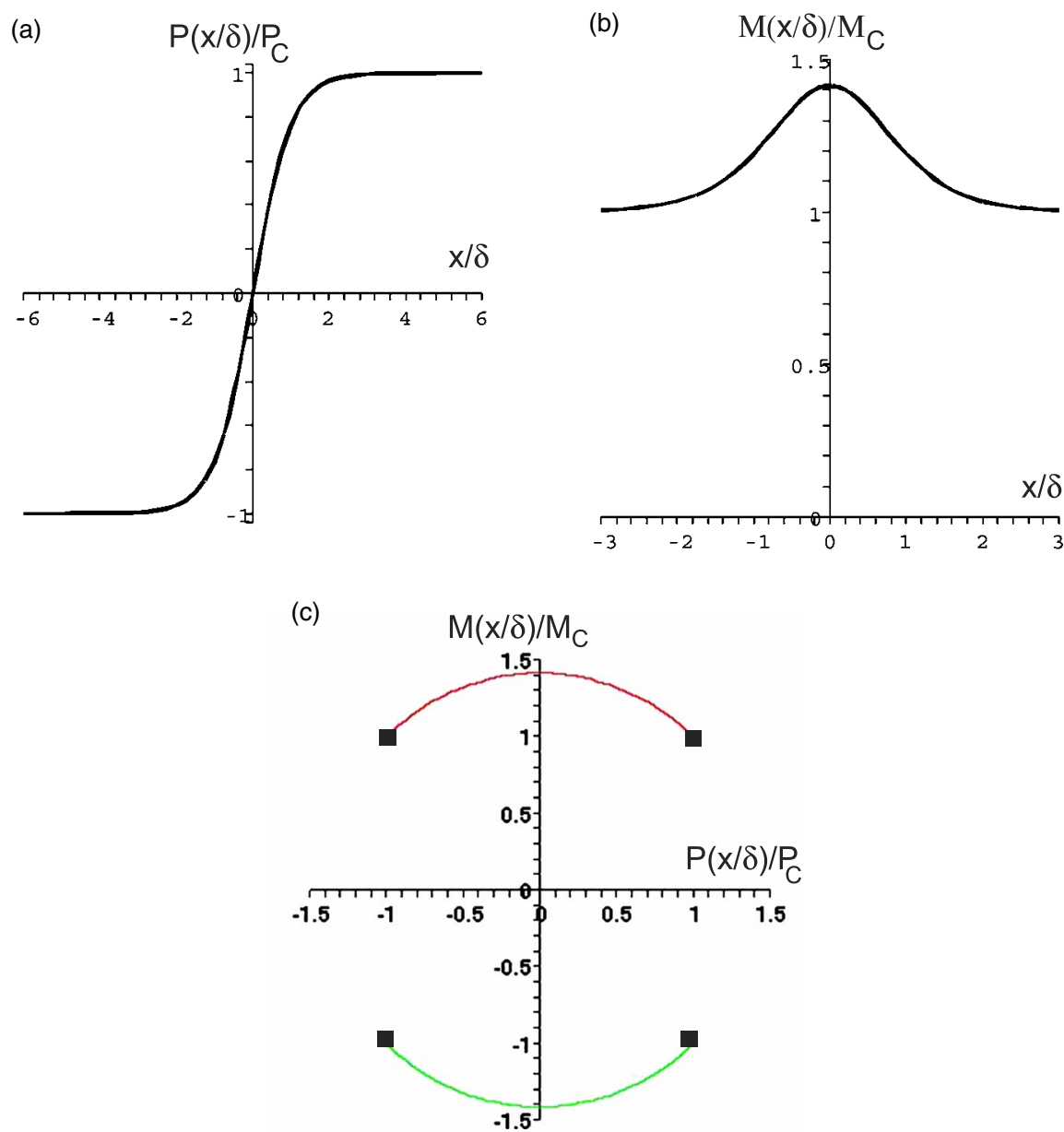

FIG. 4. (Color online) Polarization-magnetization domain wall profiles in the stability field of the low-symmetry phase with $M_{B}$ and $P_{B}$ [(a) and (b)] $P(x)$ is inverted while $M(x)$ is not. $M$ is different from zero in domains. It is different from zero in domain walls. The minima of $\mathfrak{I}_{M P}(x)$ are denoted by squares.

macroscopic magnetization in the low-symmetry $\left(P_{B}, M_{B}\right)$ phase; this is dramatically different from the scenario in the low-symmetry $\left(P_{0}, 0\right)$ phase [see Fig. 1(b)], where the domain walls carry out the total magnetization of $\mathrm{BiFeO}_{3}$ as the stripe domains are not magnetic but ferroelectric [see Fig. $1(\mathrm{a})]$.

Equation (18) shows that the maximum enhancement of magnetization that may be expected in the latter case is $M($ wall $)=\sqrt{2} M($ domain $)$. Given that the canting moment of $\mathrm{BiFeO}_{3}$ is approximately $8 \mathrm{emu} / \mathrm{cm}^{3}$, that would lead to a maximum moment of $\sim 11 \mathrm{emu} / \mathrm{cm}^{3}$ inside the wall, in excellent agreement with recent $a b$ initio calculations by Lubk et al., ${ }^{44}$ who predict a $33 \%$ increase in the canting angle, suggesting that the intrinsic magnetization of the wall may indeed be larger than that of the domain. Nevertheless, once averaged by the volume fraction of domain wall material, this enhancement is too small to account by itself for the total magnetic moment observed in thin films.

\section{CONCLUSIONS}

The present paper was intended to stimulate further work on multiferroics such as $\mathrm{BiFeO}_{3}$ and to suggest a mechanism for weak ferromagnetism in thin films that might reconcile previous disparate results. Although it is not justified to extract exact numerical conclusions in the case of $\mathrm{BiFeO}_{3}$ from our simplified free energy, it is nevertheless encouraging to show that plausible results are obtained which are quantitatively within the right order of magnitude. This may provide motivation for more realistic free-energy models in the future, as in Refs. 18 and 19, once more detailed data are obtained for bismuth ferrite. However, we stress that using a more complicated free energy, as done in Refs. 18 and 19, introduces many unknown parameters and is generally intractable analytically so that only numerical methods can be deployed.

The key predictions can be summarized as follows: (i) when a multiferroic system is in the ferroelectric state, but still above the temperature of the ferromagnetic one, it can display magnetism inside the domain wall (Figs. 1 and 2). (ii) When a multiferroic system is below both critical temperatures, the domain walls where only one of the order parameters is inverted can show an enhancement of the other, e.g., a ferroelectric wall can show enhanced magnetization (see Fig. 4). (iii) The thickness of the domain walls is affected by the coupling so that ferroelectric domain walls in multiferroic systems are thicker than usual, being intermedi- 
ate between the thickness of a purely feroelectric wall and that of a purely magnetic one.

As regards the experimentally verifiable consequences of this model, the chief experimental prediction is that an enhanced magnetization may appear in the middle of the ferroelectric domain wall. Direct measurements of domain wall properties, however, are not easy due to their reduced thickness, meaning that the total volume of magnetic material in a single wall could be below the detection limit of techniques such as magnetic force microscopy - though other microscopy techniques such as electron holography may, in principle, still work. A simpler approach, however, would be to measure the collective response of systems with many domain walls, i.e., systems with very small domains such as thin films. A first and simplest test would be to correlate the magnetization of the film and the average size of its spontaneous domains: the smaller the domain size, the bigger the magnetization. This has already been reported for thin films of $\mathrm{TbMnO}_{3} \cdot{ }^{13} \mathrm{~A}$ second test would consist in "wiping out" the domain walls by inducing a monodomain state. In a ferroelectric, this can be achieved by application of an external electric field bigger than the coercive one. Comparison of magnetization before and after removal of the domain walls should help determine their role.

Since thickness, strain, grain size and, above all, impurity levels, can also strongly affect to what extent the $\mathrm{BiFeO}_{3}$ films are antiferromagnetic or weakly ferromagnetic to start with, they will also affect which of the different discussed scenarios is relevant and thus also whether the domain wall magnetization is the key issue. We emphasize that our model does not exclude other mechanisms, and the existence of multidomain samples without enhanced magnetization stresses the importance of taking into account the specific type of wall. Likewise, whether the transition can be described by a second-order potential (as in epitaxial thin films) or first order (as in bulk), or even some intermediate, tricritical state (not considered here) also affects the predicted behavior of the magnetization. Once again, the specific circumstances of the samples are critical and may explain the difference both within and between samples made in different laboratories. As the main take-home messages regarding specifically bismuth ferrite, then, we would emphasize that (i) domain wall magnetization can in theory be big enough to measurably increase the average magnetic moment of thin films and (ii) whether or not they account for the magnetization of specific samples will depend strongly on the specific characteristics of the samples: the symmetry of the walls on one hand, and the existence of other defects such as impurities or grain boundaries on the other.

\section{APPENDIX: LANDAU COEFFICIENTS OF $\mathrm{BiFeO}_{3}$}

We provide below estimates of the Landau coefficients for $\mathrm{BiFeO}_{3}$. The minimization of $G_{M P}$ with respect to $P$ yields $\alpha+\beta P^{2}+\eta P^{4}+\gamma M^{2}=0$. Then, the equilibrium value of $P$ in the bulk yields

$$
P^{2}=\frac{-\beta+\sqrt{\beta^{2}-4 \eta \alpha-4 \eta \gamma M^{2}}}{2 \eta} .
$$

The inverse permittivity is the second derivative with respect to the polarization and in the ferroelectric phase it is

$$
\chi_{f}^{-1}=-\alpha+2 \beta P+4 \eta P^{3}+\gamma M^{2} .
$$

In order to calculate the coefficients in these expressions, we must compare with experimental results. In order to facilitate this comparison, we shall make a series of assumptions and discuss their validity.

First, although the ferroelectric phase transition in bulk $\mathrm{BiFeO}_{3}$ is first order, ${ }^{5,25,26}$ it is common for the transitions of ferroelectrics to become second order when the samples are epitaxially clamped thin films. ${ }^{22}$ In these conditions, the effective coefficient of the $P^{4}$ term, $\beta$, is positive while $\eta P^{6}$, which is much smaller in absolute value, can be discarded. The second-order approximation has been used before to describe thin films of $\mathrm{BiFeO}_{3} \cdot{ }^{23,24}$ The second approximation that we shall make is that the biquadratic magnetoelectric coupling is relatively small so that it can be treated as a perturbative contribution to the bare ferroelectric solution.

Starting with Eq. (A1), and making the simplifications mentioned above (second-order approach, small magnetoelectric coupling), the polarization of a simple ferroelectric is $P_{0}^{2}=-\frac{\alpha}{\beta}$ while the solution including the magnetoelectric coupling is $P^{2} \approx P_{0}^{2}-\frac{\gamma}{\beta} M^{2}$. The magnetoelectric polarization is usually much smaller than the ferroelectric one so a firstorder Taylor expansion yields $P \approx P_{0}-M^{2} \gamma /\left(2 \beta P_{0}\right)$. Meanwhile, the inverse ferroelectric permittivity is [Eq. (A2)] $\chi_{f}^{-1}=-\alpha+2 \beta P+\gamma M^{2} \approx 2 \beta P_{0}^{2}$. Finally, the inverse magnetic susceptibility is found by differentiating the free energy with respect to $M$. For the phase $\left(P=P_{0}, M=0\right)$, this is $\chi_{M}^{-1}=a$ $+\gamma P_{0}^{2} \approx a$. Thus, the $\alpha, \beta, \gamma$, and $a$ coefficients can be extracted from the bulk permittivity, susceptibility, and polarization.

The dielectric constant of $\mathrm{BiFeO}_{3}$ at room temperature is $\chi_{f} \sim 30 \varepsilon_{0}$ (Ref. 25) and the polarization of goodquality single crystals is around $P_{0} \sim 1 \mathrm{C} \mathrm{m}^{-2}, 45,46$ hence $\alpha \sim-1.7 \times 10^{9} \mathrm{C}^{-2} \mathrm{~m}^{2} \mathrm{~N}, \quad \beta \sim 1.7 \times 10^{9} \mathrm{C}^{-4} \mathrm{~m}^{6} \mathrm{~N} \quad$ (S.I.). The magnetic susceptibility of $\mathrm{BiFeO}_{3}$ is $\chi_{M}=0.6$ $\times 10^{-5} \mathrm{~g}^{-1}$ emu Oe ${ }^{-1}=4.7 \times 10^{-5} \mathrm{C}^{-2} \mathrm{~m} \mathrm{~kg}$ so that the firstorder Landau coefficient is $a \sim 2.1 \times 10^{4} \mathrm{C}^{2} \mathrm{~m}^{-1} \mathrm{~kg}^{-1}$.

As for the magnetoelectric coefficient, simple arithmetic shows that $P$ [i.e., $\left.P=P_{0}-M^{2} \gamma /\left(2 \beta P_{0}\right)\right]$ expressed in terms of $\chi_{f}^{-1}$ (i.e., $\chi_{f}^{-1}=2 \beta P_{0}^{2}$ ) is $P=P_{0}+\gamma \chi_{f} P_{0} M^{2}$ and the magnetoelectric polarization is therefore, $P_{M E} \approx \gamma \chi_{f} P_{0} M^{2}$. $\mathrm{BiFeO}_{3}$ has no net magnetization in the absence of magnetic fields (the small canting moment is cancelled by a long-period spin cycloid), and the magnetization is essentially proportional to the external magnetic field, so $M=\chi_{M} H$ and $P_{M E}$ $\approx \gamma \chi_{f} P_{0} \chi_{M}^{2} H^{2}$. Thus, the magnetoelectric polarization can be calculated from the $P$-vs- $H$ curves reported in Ref. 43. From these, one finds $P_{M E} \approx \zeta H^{2}$ for low magnetic fields, where $\zeta=4.4 \times 10^{-8} \mathrm{C} \mathrm{m}^{-2} \mathrm{~T}^{-2}=7 \times 10^{-20} \mathrm{C} \mathrm{A}^{-2}$. Supplying these values in $\zeta$ allows us to calculate the magnetoelectric coupling coefficient $\gamma=\zeta \chi_{f}^{-1} \chi_{M}^{-2} P_{0}^{-1}=0.12 \mathrm{~m} \mathrm{~kg}^{-1}$.

We also need to calculate the gradient energy coefficients, $\lambda$ and $\kappa$. Referring to Ref. 47 and the value of $\chi_{M}^{-1}$ reported above, we get $\lambda=3 \times 10^{-12} \mathrm{C}^{2} \mathrm{~kg}^{-1} \mathrm{~m}$. The value of the ferroelectric "exchange constant" $\kappa$ is not known exactly for $\mathrm{BiFeO}_{3}$ but we assume a value of $\kappa \sim 1$ $\times 10^{-10} \mathrm{C}^{-2} \mathrm{~m}^{5} \mathrm{~kg} \mathrm{~s}^{-2}$, which is typical for other perovskite ferroelectrics, although the very high $T_{\mathrm{c}}$ of $\mathrm{BiFeO}_{3}$ probably 
means that this is probably an underestimate ( $k$ is a measure of the dipole-dipole interaction, which is expected to be stronger in ferroelectrics that have high ordering temperatures).

Other sources of uncertainty are the value of $a \sim \chi_{M}^{-1}$, which has been calculated by neglecting the higher-order terms in the magnetic susceptibility, and the value of $\eta$. For the latter, the only value reported in the literature is $\eta=6$ $\times 10^{6} \mathrm{~m}^{9} \mathrm{C}^{4} \mathrm{~F}$ (S.I.). Note that this is approximately three orders of magnitude lower than $\beta^{2} / 4 / \alpha$ in our set of coefficients but the difference is smaller if comparing to the other coefficients in Ref. 24. If $\eta$ were bigger than $\beta^{2} / 4 \alpha$ this could double or triple the value of $M_{0}$ (e.g., $M_{0}$ $=4830 \mathrm{~A} / \mathrm{m}$ and $\left.M_{D W}=20 \mathrm{emu} / \mathrm{cm}^{3}\right)$ so that the $M_{0}$ calculated using these coefficients $\left(M_{0}=1.3 \times 10^{5} \mathrm{~A} / \mathrm{m}, M_{D W}\right.$ $\sim 7800 \mathrm{~A} / \mathrm{m})$ can in fact be considered as a conservative estimate. The complete set of Landau coefficients used for our calculations is summarized in the table below (S.I. units)

\begin{tabular}{ccccccc}
$\alpha$ & $\beta$ & $\eta$ & $\gamma$ & $\kappa$ & $\lambda$ & $a$ \\
\hline$-1.7 \times 10^{9}$ & $1.7 \times 10^{9}$ & $6 \times 10^{6}$ & 0.12 & $1 \times 10^{-10}$ & $3 \times 10^{-12}$ & $2.1 \times 10^{4}$
\end{tabular}

*maren.daraktchiev@dh.gsi.gov.uk

† gustau.catalan@cin2.es

\#jfs32@hermes.cam.ac.uk

${ }^{1}$ J. Wang, J. N. Neaton, H. Zheng, V. Nagarajan, S. B. Ogale, B. Liu, D. Viehland, V. Vaithyanathan, D. G. Schlom, U. V. Waghmare, N. A. Spaldin, K. M. Rabe, M. Wuttig, and R. Ramesh, Science 299, 1719 (2003).

${ }^{2}$ P. Fischer, M. Polomska, I. Sosnowska, and M. Szymanski, J. Phys. C 13, 1931 (1980).

${ }^{3}$ G. A. Smolenskii, V. A. Isupov, A. Agranowskaya, and N. Krainik, Sov. Phys. Solid State 2, 2651 (1961).

${ }^{4}$ S. Kiselev, R. Ozerov, and G. Zhdanov, Sov. Phys. Dokl. 7, 742 (1963).

${ }^{5}$ G. Catalan and J. F. Scott, Adv. Mater. 21, 2463 (2009).

${ }^{6}$ W. Eerenstein, F. Morrison, J. Dho, M. Blamire, J. Scott, and N. Mathur, Science 307, 1203a (2005).

${ }^{7}$ H. Béa, M. Bibes, A. Barthélémy, K. Bouzehouane, E. Jacquet, A. Khodan, J.-P. Contour, S. Fusil, F. Wyczisk, A. Forget, D. Lebeugle, D. Colson, and M. Viret, Appl. Phys. Lett. 87, 072508 (2005).

${ }^{8}$ X. J. Lou, C. X. Yang, T. A. Tang, Y. Y. Lin, M. Zhang, and J. F. Scott, Appl. Phys. Lett. 90, 262908 (2007).

${ }^{9}$ B. Houchmandzadeh, J. Lajzerowicz, and E. Salje, J. Phys.: Condens. Matter 3, 5163 (1991).

${ }^{10}$ J. Přívratská and V. Janovec, Ferroelectrics 204, 321 (1997).

${ }^{11}$ A. Goltsev, R. Pisarev, T. Lottermoser, and M. Fiebig, Phys. Rev. Lett. 90, 177204 (2003).

${ }^{12}$ T. Lottermoser and M. Fiebig, Phys. Rev. B 70, 220407(R) (2004).

${ }^{13}$ C. Daumont, Ph.D. thesis, University of Groningen, 2009.

${ }^{14}$ A. S. Logginov, G. A. Meshkov, A. V. Nikolaev, E. P. Nikolaeva, A. P. Pyatakov, and A. K. Zvezdin, Appl. Phys. Lett. 93, 182510 (2008).

${ }^{15}$ G. Catalan, H. Béa, S. Fusil, M. Bibes, P. Paruch, A. Barthélémy, and J. F. Scott, Phys. Rev. Lett. 100, 027602 (2008).

${ }^{16}$ G. Catalan, J. F. Scott, A. Schilling, and J. M. Gregg, J. Phys.: Condens. Matter 19, 022201 (2007).

${ }^{17}$ M. Daraktchiev, G. Catalan, and J. F. Scott, Ferroelectrics 375, 122 (2008)
${ }^{18}$ D. L. Fox, D. R. Tilley, J. F. Scott, and H. J. Guggenheim, Phys. Rev. B 21, 2926 (1980).

${ }^{19}$ D. R. Tilley and J. F. Scott, Phys. Rev. B 25, 3251 (1982).

${ }^{20}$ D. L. Fox and J. F. Scott, J. Phys. C 10, L329 (1977).

${ }^{21}$ W. F. Brown, R. M. Hornreich, and S. Shtrikman, Phys. Rev. 168, 574 (1968).

${ }^{22}$ J. F. Scott, Phase Transitions 30, 107 (1991).

${ }^{23}$ J. X. Zhang, Y. L. Li, Y. Wang, Z. K. Liu, L. Q. Chen, Y. H. Chu, F. Zavaliche, and R. Ramesh, J. Appl. Phys. 101, 114105 (2007).

${ }^{24}$ Q. Jiang and J. H. Qiu, J. Appl. Phys. 99, 103901 (2006).

${ }^{25}$ S. Kamba, D. Nuzhnyy, M. Savinov, J. Sebek, J. Petzelt, J. Prokleska, R. Haumont, and J. Kreisel, Phys. Rev. B 75, 024403 (2007).

${ }^{26}$ D. C. Arnold, K. S. Knight, F. D. Morrison, and P. Lightfoot, Phys. Rev. Lett. 102, 027602 (2009).

${ }^{27}$ M. Mostovoy, Phys. Rev. Lett. 96, 067601 (2006).

${ }^{28}$ R. Rajaraman, Phys. Rev. Lett. 42, 200 (1979).

${ }^{29}$ C. Montonen, Nucl. Phys. B 112, 349 (1976).

${ }^{30}$ E. M. Lifshitz, Zh. Eksp. Teor. Fiz. 19, 353 (1944).

${ }^{31}$ R. Pirc and R. Blinc, Phys. Rev. B 60, 13470 (1999).

${ }^{32}$ J. Seidel, L. W. Martin, Q. He, Q. Zhan, Y.-H. Chu, A. Rother, M. E. Hawkridge, P. Maksymovych, P. Yu, M. Gajek, N. Balke, S. V. Kalinin, S. Gemming, H. Lichte, F. Wang, G. Catalan, J. F. Scott, N. A. Spaldin, J. Orenstein, and R. Ramesh, Nature Mater. 8, 229 (2009).

${ }^{33}$ D. Lee, R. K. Behera, P. Wu, H. Xu, Y. L. Li, S. B. Sinnott, S. R. Phillpot, L. Q. Chen, and V. Gopalan, Phys. Rev. B 80, 060102(R) (2009).

${ }^{34}$ J.-C. Toledano and P. Toledano, The Landau Theory of Phase Transition (World Scientific, Singapore, 1987).

${ }^{35}$ Yu. M. Gufan and S. Larin, Sov. Phys. Solid State 22, 270 (1980).

${ }^{36}$ J. Holakovský, Phys. Status Solidi B 56, 615 (1973).

${ }^{37}$ R. Ramesh (private communication).

${ }^{38}$ Y. B. Chen, M. B. Katz, X. Q. Pan, R. R. Das, D. M. Kim, S. H. Baek, and C. B. Eom, Appl. Phys. Lett. 90, 072907 (2007).

${ }^{39}$ L. X. He and D. Vanderbilt, Phys. Rev. B 68, 134103 (2003).

${ }^{40}$ M. Bartels, V. Hagen, M. Burianek, M. Getzlaff, U. Bismayer, 
and R. Wiesendanger, J. Phys.: Condens. Matter 15, 957 (2003).

${ }^{41}$ A. Aird and E. K. H. Salje, Eur. Phys. J. B 15, 205 (2000).

${ }^{42}$ Z. V. Gabbasova, M. D. Kuz'min, A. K. Zvezdin, I. S. Dubenko, V. A. Murashov, D. N. Rakov, and I. B. Krynetsky, Phys. Lett. A 158, 491 (1991).

${ }^{43}$ A. Zvezdin, A. Kadomtseva, S. Krotov, A. Pyatakov, Y. Popov, and G. Vorob'ev, J. Magn. Magn. Mater. 300, 224 (2006).
${ }^{44}$ A. Lubk, S. Gemming, and N. A. Spaldin, Phys. Rev. B 80, 104110 (2009).

${ }^{45}$ M. Cazayous, D. Malka, D. Lebeugle, and D. Colson, Appl. Phys. Lett. 91, 071910 (2007).

${ }^{46}$ D. Lebeugle, D. Colson, A. Forget, M. Viret, P. Bonville, J. Marucco, and S. Fusil, Phys. Rev. B 76, 024116 (2007).

${ }^{47}$ A. Zvezdin and A. Pyatakov, Phys. Usp. 47, 416 (2004). 